\title{
Japan's Advanced Medical Care Program for rapid introduction of unevaluated healthcare technologies toward National Health Insurance (NHI) coverage
}

\author{
Shoji Sanada ${ }^{1}$, Keiko Ueda ${ }^{2}$, Naoto Uemura ${ }^{1,3}$ \\ ${ }^{1}$ Department of Medical Innovation, Osaka University Hospital, Japan, ${ }^{2}$ Clinical Research Support Center, The \\ University of Tokyo, Japan, ${ }^{3}$ Department of Clinical Pharmacology and Therapeutics, Oita University Faculty of \\ Medicine, Japan
}

Backgrounds: Advanced Medical Care Program (AMCP) is a unique program designated in Japan's National Health Insurance (NHI) system to facilitate patients' access to novel but promising healthcare technologies with governmental assessments toward NHI coverage. While any reimbursement of medical fees for a series of medical care including the use of any unapproved technology (medicine, medical device or surgical procedure) in part is primarily not allowed by the NHI system in Japan, AMCP Type-B exceptionally allows clinical use of unapproved technologies in the NHI system under mixed billing comprising of total billing on patients for unapproved segments combined with the rest reimbursed by NHI. The present study examined how AMCP contributes to accelerated introduction of new technologies into the NHI coverage.

Methods: To access the relationship between AMCP and NHI coverage, we systematically reviewed all AMCP type-B technologies submitted to the AMCP Technical Review Board of Ministry of Health, Labour and Welfare (MHLW) in Japan, from May, 2006 to July, 2017. The data such as Board Meeting minutes and publicized documents were compiled from AMCP web database. We additionally integrated all the data from CSRs or review reports from the Japanese regulatory authority (Pharmaceuticals and Medical Devices Agency) and the current NHI fee table, to confirm the final status (continued/finished/approved/abandoned) for each AMCP technology.

Results: Of 95 novel, unapproved technologies submitted as AMCP type-B, 38 were completed. Of them, 26 underwent final reviews of the Board with respect to their safety and efficacy. Nine were denied due to lack of efficacy and 7 were recognized as therapeutics of demonstrated effectiveness (3 surgical procedures, 2 medical devices and 2 pharmaceuticals). These 7 technologies were successfully introduced to the NHI coverage. One orphan drug and 2 devices were reviewed through the accelerated frameworks for market approval.

Discussion/Conclusion: This is the first attempt to comprehensively capture all the information regarding AMCPs submitted to the AMCP Technical Review Board and their outcomes with respect to NHI coverage. Strategic approach with careful selection of target therapeutics is the key to warrant a rapid introduction of therapeutics through AMCP. 